\title{
AVALIAÇÃO DO COMPORTAMENTO DA ADIÇÃO DE VANÁdIO E POTÁSSIO SOBRE ALUMINA DE TRANSIÇÃO
}

\author{
M. S. P. MARTINS ${ }^{1}$, J. A. J. RODRIGUES ${ }^{2}$ e G. G. CORTEZ ${ }^{1}$ \\ ${ }^{1}$ Escola de Engenharia de Lorena - USP - Departamento de Engenharia Química \\ ${ }^{2}$ Instituto Nacional de Pesquisas Espaciais-LCP \\ E-mail para contato: cortez@dequi.eel.usp.br
}

RESUMO - Aluminas de transição são muito utilizadas como suportes de catalisadores. Pela reação de decomposição do isopropanol notou-se que a adição de vanádio e potássio no suporte modificou a formação de propeno devido à diminuição de sítios ácidos sobre o suporte.

\section{INTRODUÇÃO}

O uso da $\gamma$-alumina como suporte de catalisadores tem sido extensivamente investigado em diversos processos industriais, principalmente os petroquímicos (Juan-Juan et al., 2006).

Óxidos metálicos do grupo V suportados em $\gamma$-alumina têm uma grande variedade em aplicações catalíticas (Cortez et al., 2003). Os óxidos metálicos suportados apresentam uma melhor estabilidade térmica das estruturas do óxido do metal e uma área superficial maior (Scholz et al., 2013). A adição de potássio ao suporte de alumina impregnado com vanádio diminui a atividade, porém aumenta a seletividade à propeno (Ermini et al., 2000).

Este estudo tem como objetivos sintetizar um hidróxido de alumínio e calciná-lo a diferentes temperaturas para ser utilizado como suporte; preparar catalisadores suportados de vanádio e potássio; caracterizar os suportes e catalisadores através das técnicas de volumetria de $\mathrm{N}_{2}$, redução à temperatura programada (RTP) e difratometria de raios X (DRX) e avaliar as propriedades ácidas e/ou básicas do catalisador através da reação de decomposição do isopropanol.

\section{EXPERIMENTAL}

\subsection{Síntese dos catalisadores}

Sintetizou-se o precursor através do método de precipitação com o uso de uma solução de $\mathrm{AlCl}_{3}$ e uma solução de $\mathrm{NaOH}$. Adicionou-se as duas soluções em um reator batelada sob agitação mecânica. Realizou-se a lavagem da amostra e então deixou-a por uma noite na estufa. Esse material foi calcinado até as temperaturas finais de 450 e $500{ }^{\circ} \mathrm{C}$, obtendo-se o suporte identificado por $\mathrm{Al}_{2} \mathrm{O}_{3}$-X, onde $\mathrm{x}$ representa a temperatura de calcinação. Em seguida, prepararam-se duas séries de catalisadores. A primeira série foi preparada impregnando-se os suportes com 4 átomos de vanádio/nm² de alumina. A mistura da solução aquosa de $\mathrm{NH}_{4} \mathrm{VO}_{3}$ e o suporte na forma de pó foi realizada em um roto-evaporador a vácuo. Após a etapa de impregnação, o pó residual foi seco em estufa e depois calcinado até as temperaturas de 450 e 
$500{ }^{\circ} \mathrm{C}$, obtendo-se assim os catalisadores identificados por V/Al-x. Para se obter a segunda série de catalisadores, o suporte foi co-impregnado com uma quantidade total de 8 átomos $(\mathrm{V}+\mathrm{K})$ por $\mathrm{nm}^{2}$ de alumina. Para isso, utilizou-se soluções aquosas de $\mathrm{KOH}$ e $\mathrm{NH}_{4} \mathrm{VO}_{3}$ e, pelo mesmo método descrito acima, obteve-se os catalisadores identificados por VK/Al-x. Os catalisadores foram caracterizados pelas técnicas de volumetria de $\mathrm{N}_{2}$, DRX e RTP e pela reação de decomposição do isopropanol.

\subsection{Caracterização físico-química}

As medidas de áreas específicas foram determinadas a partir das isotermas de adsorçãodessorção de $\mathrm{N}_{2}$ a $-196{ }^{\circ} \mathrm{C}$, usando-se o equipamento Belsorp II - mini.

As análises de DRX foram realizadas utilizando-se o método do pó, através do equipamento da marca Panalytical, modelo Enpyrean. As fases cristalinas foram identificadas através do programa graph analyzer criado pelo INPE de Cachoeira Paulista.

As análises de RTP dos catalisadores suportados foram realizadas em um equipamento Quantachrome, modelo Chembet-3000, equipado com um detector de condutividade térmica.

As propriedades ácidas e/ou básicas dos catalisadores foram avaliadas através da reação de decomposição do isopropanol. O reator foi alimentado com isopropanol que foi injetado na tubulação do reator com o auxílio de uma bomba (Thermo Separation Products, modelo P100) e diluído em hélio. Os gases efluentes do reator foram analisados por cromatografia gasosa, utilizando-se um cromatógrafo da marca Varian, modelo 3380, equipado com um detector de condutividade térmica (DCT), e conectado em linha com o sistema reacional através de uma válvula de injeção automática. Para separação e análise dos efluentes do reator foi utilizada uma coluna de aço inox empacotada com fase estacionária Poropak-Q $(4,5 \mathrm{~m})$. A reação foi avaliada um tempo de residência $\left(\mathrm{W} / \mathrm{F}_{\mathrm{A} 0}\right)$ igual a $6,3 \mathrm{~g} \cdot \mathrm{h} / \mathrm{mol}$. A taxa específica de formação dos produtos (TEP) foi calculada a partir das equações abaixo:

$$
T E P=\frac{S_{P} \times T E R}{S_{B E T}}
$$

$$
T E R=\frac{X_{A} \times F_{A 0}}{W \times S_{B E T}}
$$

$$
\begin{aligned}
& X_{A}(\%)=\left(\frac{n_{R}}{n_{R_{0}}}\right) \times 100 \\
& S_{P}(\%)=\left(\frac{n_{R}}{n_{R_{0}}}\right) \times\left(\frac{N_{C P}}{N_{C R}}\right) \times 100,
\end{aligned}
$$

onde TER é a taxa específica de reação, $\mathrm{X}_{\mathrm{A}}$ é a conversão do isopropanol, $\mathrm{S}_{\mathrm{P}} \mathrm{a}$ 
seletividade dos produtos, $n_{R}$ é o número de mols do isopropanol consumido, ${ }_{R}$ é o número de mols de isopropanol na alimentação e ${ }^{N_{C P}}$ e ${ }^{N_{C R}}$ são os números de átomos de carbono presentes nos produtos formados e no isopropanol, respectivamente.

\section{RESULTADOS E DISCUSSÕES}

As áreas específicas $\left(\mathrm{S}_{\mathrm{BET}}\right)$ e o volume total de poros $(\mathrm{Vp})$ dos suportes e catalisadores são apresentados na Tabela 1. Comparando-se o suporte $\mathrm{Al}_{2} \mathrm{O}_{3}$-X com os catalisadores V/Al-X e VK/Al-x observou-se que a impregnação da alumina com vanádio, nas duas temperaturas de calcinação, gerou uma diminuição no valor da área específica. Na impregnação de vanádio e potássio sobre o suporte pelo método da co-impreganação, notou-se também uma diminuição da área específica. Ao se comparar os suportes e os catalisadores calcinados a $450^{\circ} \mathrm{C}$ com os calcinados a $500^{\circ} \mathrm{C}$, observou-se que o aumento na temperatura de calcinação causou também uma diminuição na área específica. Por outro lado, observou-se uma pequena variação no volume de poros dos catalisadores V/Al-x e VK/Al-x, quando comparados entre si. Uma maior variação foi visualizada quando se comparou os suportes com os dois catalisadores.

Tabela 1 - Valores de área específica $\left(\mathrm{S}_{\mathrm{BET}}\right)$ e volume de poros $(\mathrm{Vp})$.

\begin{tabular}{ccccc}
\hline Catalisadores & $\mathrm{V}_{2} \mathrm{O}_{5}(\% \mathrm{p} / \mathrm{p})$ & $\mathrm{K}_{2} \mathrm{O}(\% \mathrm{p} / \mathrm{p})$ & $\mathrm{S}_{\mathrm{g}}\left(\mathrm{m}^{2} / \mathrm{g}\right)$ & $\mathrm{Vp}\left(\mathrm{cm}^{3} / \mathrm{g}\right)$ \\
\hline $\mathrm{Al}_{2} \mathrm{O}_{3}-450{ }^{\circ} \mathrm{C}$ & - & - & 263 & 0,37 \\
$\mathrm{~V} / \mathrm{Al}-450{ }^{\circ} \mathrm{C}$ & 24,1 & - & 243 & 0,30 \\
$\mathrm{VK} / \mathrm{Al}-450{ }^{\circ} \mathrm{C}$ & 24,1 & 2,0 & 239 & 0,29 \\
\hline $\mathrm{Al}_{2} \mathrm{O}_{3}-500{ }^{\circ} \mathrm{C}$ & - & - & 215 & 0,38 \\
$\mathrm{~V} / \mathrm{Al}-500^{\circ} \mathrm{C}$ & 20,6 & - & 206 & 0,34 \\
$\mathrm{VK} / \mathrm{Al}-500^{\circ} \mathrm{C}$ & 20,6 & 1,6 & 197 & 0,32 \\
\hline
\end{tabular}

A Figura 1 mostra as isotermas de adsorção-dessorção de $\mathrm{N}_{2}$ a $-196{ }^{\circ} \mathrm{C}$. Para todas as amostras foram observadas isotermas do tipo IV, onde o ramo inferior mostra a quantidade de nitrogênio adsorvido com o aumento da pressão relativa, enquanto que o ramo superior representa a quantidade do gás dessorvido no processo inverso. Esse tipo de isoterma é característico de sólidos mesoporosos e macroporosos (Teixeira et al., 2001).

A Figura 2 mostra a distribuição do diâmetro de poros e do volume de poros. Os catalisadores e os suportes apresentaram um comportamento monomodal, com distribuição de poros na faixa de 0 a $10 \mathrm{~nm}$. $\mathrm{O}$ aumento da temperatura de calcinação dos catalisadores e suportes praticamente não alterou o volume de seus poros. O aumento inicial no volume de poros a baixas pressões relativas é devido a presença de microporos nos catalisadores.

As análises de RTP dos catalisadores são apresentadas na Figura 3. O óxido de vanádio apresenta três temperaturas máximas de redução a 655,690 e $823{ }^{\circ} \mathrm{C}$, que corresponde a seguinte etapa de redução das espécies de vanádio, descrito por Korane et al. (1994):

$$
\mathrm{V}_{2} \mathrm{O}_{5} \rightarrow \mathrm{V}_{6} \mathrm{O}_{13}\left(675^{\circ} \mathrm{C}\right) ; \mathrm{V}_{6} \mathrm{O}_{13} \rightarrow \mathrm{V}_{2} \mathrm{O}_{4}\left(705^{\circ} \mathrm{C}\right) ; \mathrm{V}_{2} \mathrm{O}_{4} \rightarrow \mathrm{V}_{2} \mathrm{O}_{3}\left(780^{\circ} \mathrm{C}\right)
$$


Os catalisadores apresentaram um único pico de redução do vanádio, correspondente à redução de $\mathrm{V}_{2} \mathrm{O}_{5}$ a $\mathrm{V}_{6} \mathrm{O}_{13}$. Comparando-se os catalisadores $\mathrm{V} / \mathrm{Al}$-x com os catalisadores VK/Al-x, notou-se que a co-impregnação com potássio causou um aumento na temperatura de redução do vanádio desde 506 a $549{ }^{\circ} \mathrm{C}$, nos catalisadores calcinados a $450{ }^{\circ} \mathrm{C}$ e um aumento de 509 à $542{ }^{\circ} \mathrm{C}$, nos calcinados a $500{ }^{\circ} \mathrm{C}$. Em contrapartida, comparando-se entre si os catalisadores impregnados apenas com vanádio, notou-se que o aumento na temperatura de calcinação gerou um aumento na temperatura de redução do vanádio de 506 para $509{ }^{\circ} \mathrm{C}$. Entretanto, quando os catalisadores co-impregnados com vanádio e potássio foram comparados, notou-se que houve uma diminuição de 549 para $542{ }^{\circ} \mathrm{C}$. A diminuição da temperatura de redução do vanádio pode estar relacionada a uma interação entre o vanádio e o potássio sobre o suporte que estaria dificultando a redução do vanádio.

Figura 1- Isotermas de adsorção-dessorção dos suportes e catalisadores.
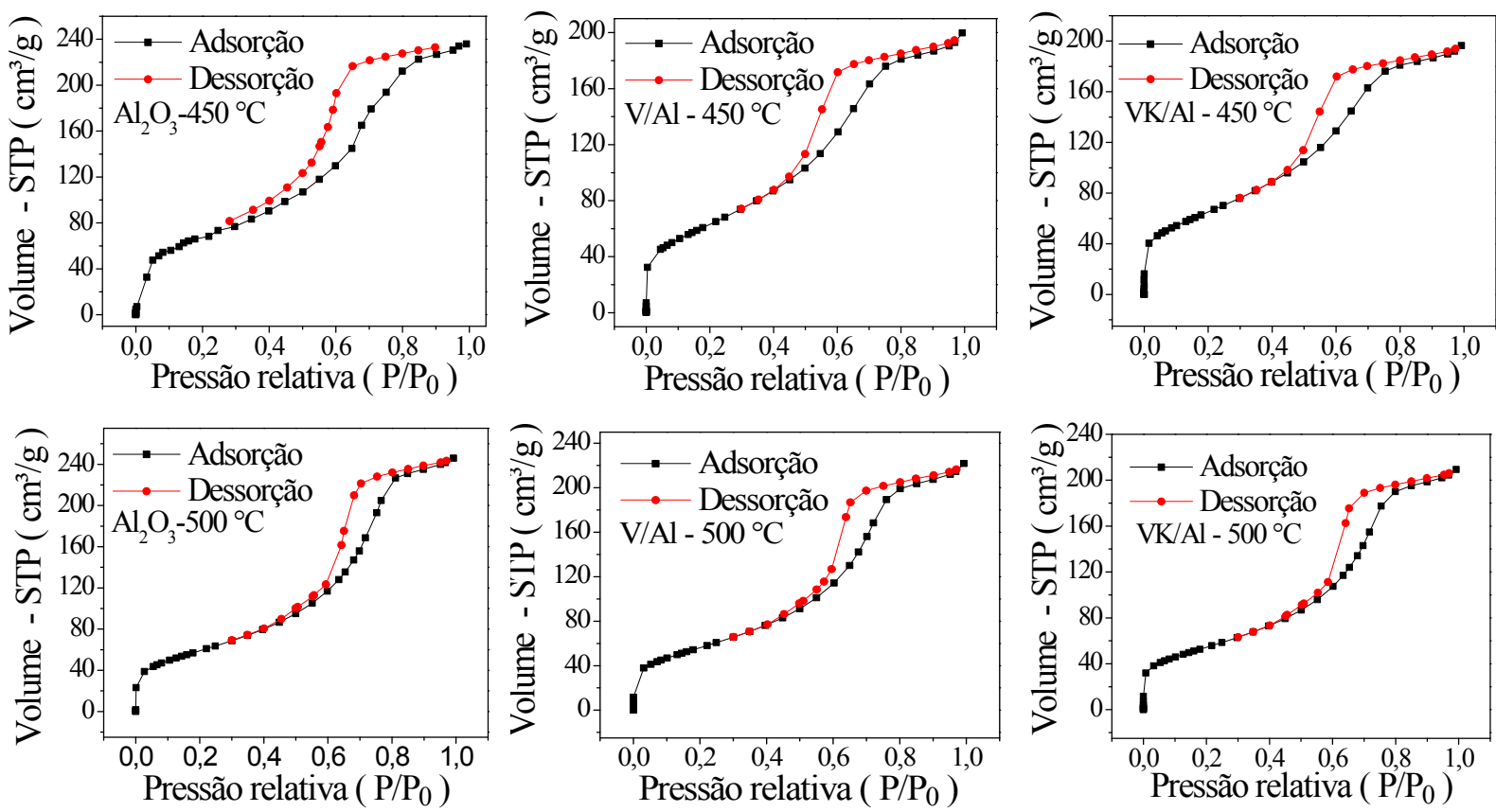

Figura 2 - Volume de poros versus diâmetro de poros para os suportes e catalisadores.
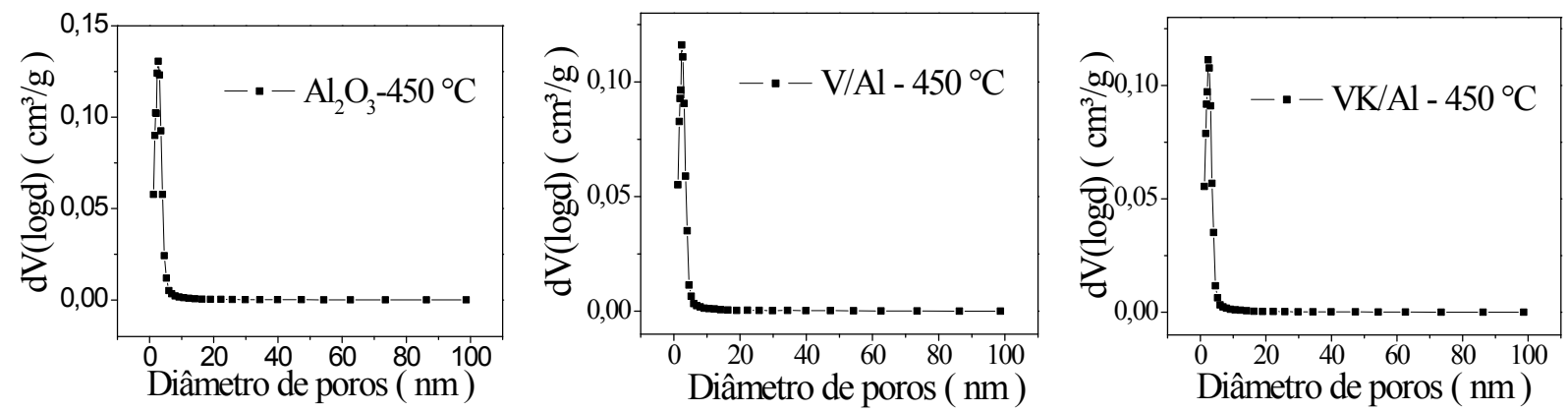

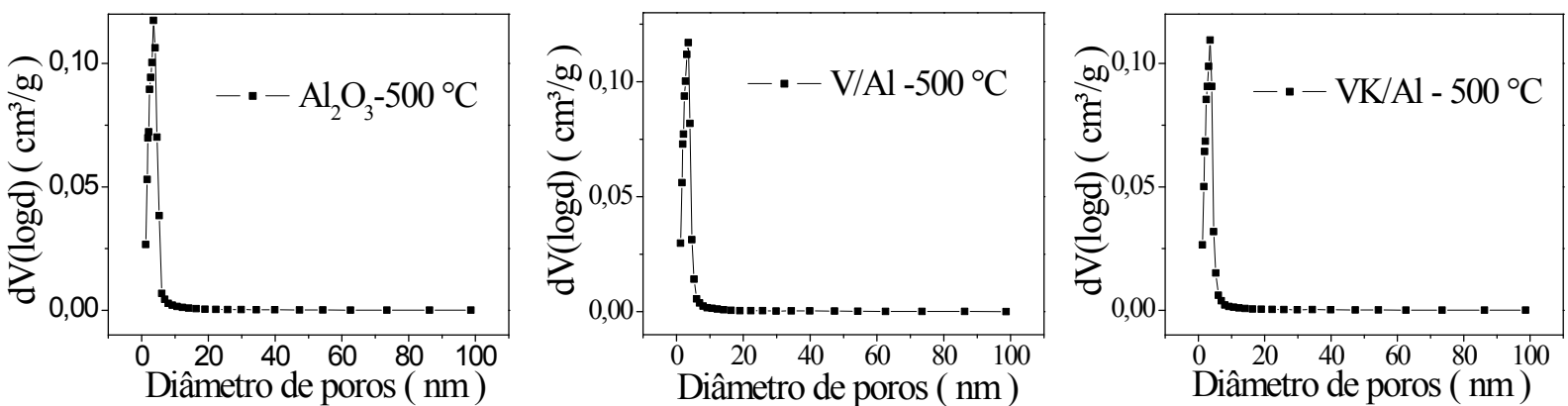

Os perfis de DRX dos suportes e catalisadores são apresentados na Figura 4. O precursor seco, preparado a partir do método da precipitação, apresentou as fases boemita, com estrutura ortorrômbica, e baierita, com estrutura monoclínica. Assim, como já era esperado, o DRX das amostras calcinadas a 450 e $500{ }^{\circ} \mathrm{C}$ comprovou a presença das aluminas de transição $\gamma-\mathrm{Al}_{2} \mathrm{O}_{3}$ e $\eta-\mathrm{Al}_{2} \mathrm{O}_{3}$, respectivamente. A impregnação com vanádio e a coimpregnação com vanádio e potássio não provocou mudanças significativas no DRX. A ausência de picos referente ao vanádio e ao potássio nas amostras impregnadas indica que seus óxidos podem estar presentes em estado amorfo, ou altamente dispersos sobre o suporte.

Figura 3 - RTP dos catalisadores.
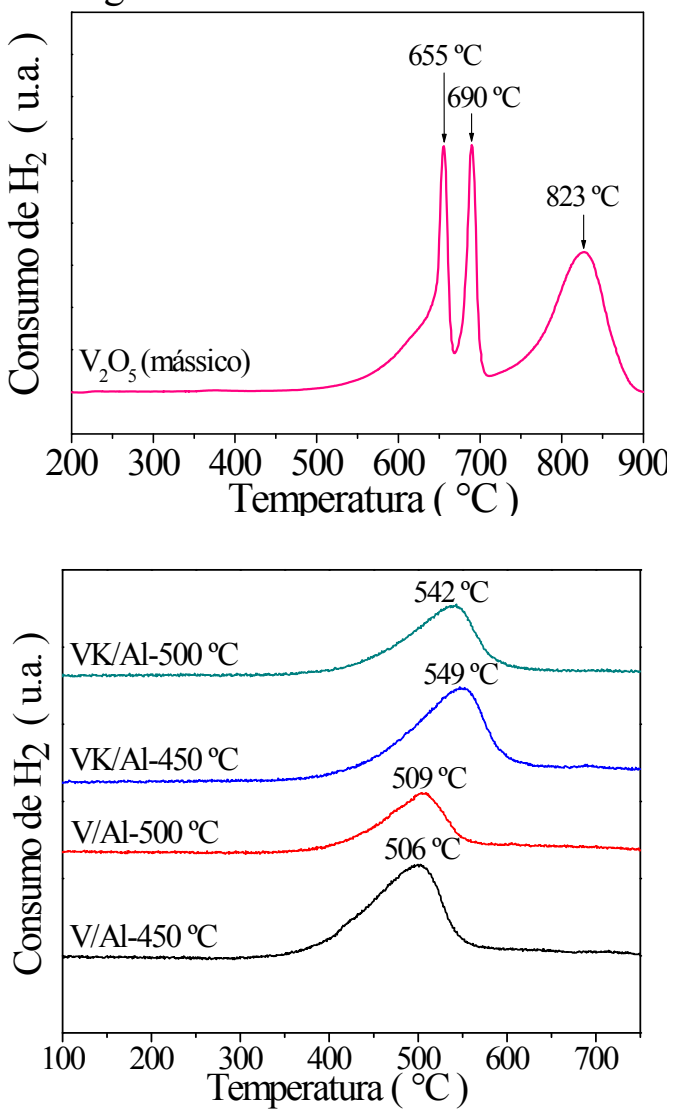

Figura 4 - DRX do precursor e das amostras calcinadas. Picos referentes às fases $(\bullet) \gamma-\mathrm{Al}_{2} \mathrm{O}_{3}$, $(\square) \eta-\mathrm{Al}_{2} \mathrm{O}_{3},\left(^{\bullet}\right)$ boemita e ( $\left.\square\right)$ baierita.

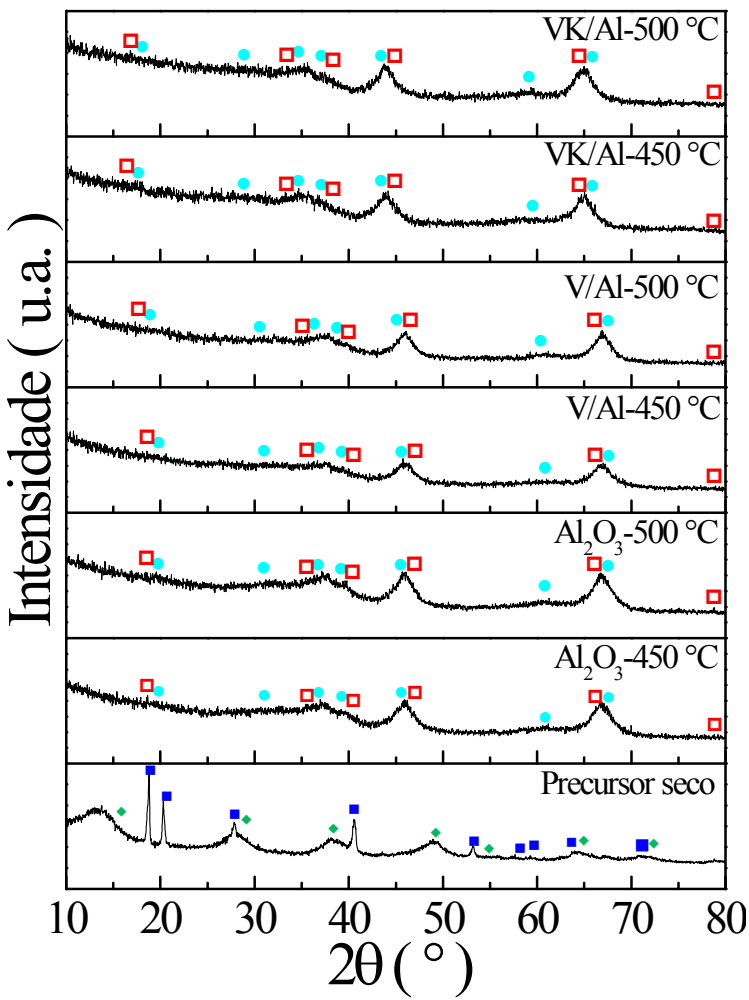

Tabela 2 - Resultados da taxa específica de reação (TER) e da taxa específica de formação dos produtos (TEP) dos catalisadores a $250{ }^{\circ} \mathrm{C}$.

Catalisadores $\begin{gathered}\text { TER } \\ \left(\mu \mathrm{mol} \cdot \mathrm{m}^{-2} \cdot \mathrm{min}^{-1}\right)\end{gathered} \quad$ TEP $\left(\mu \mathrm{mol} \cdot \mathrm{m}^{-2} \cdot \mathrm{min}^{-1}\right) / 250{ }^{\circ} \mathrm{C}$




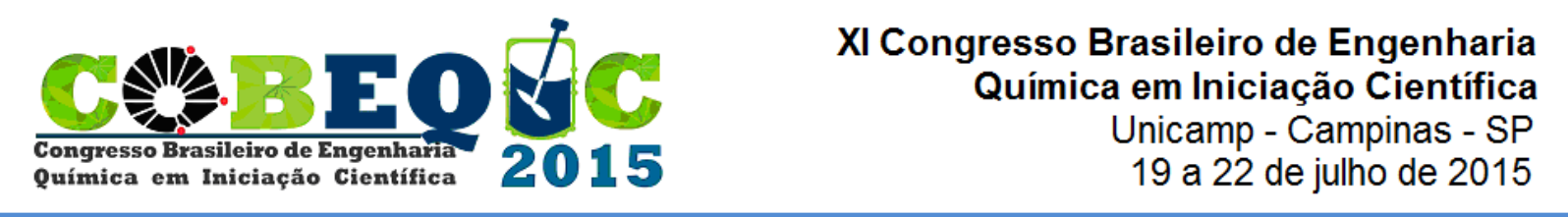

\begin{tabular}{cccc}
\hline & & Propeno & Éter diisopropílico \\
\hline $\mathrm{Al}_{2} \mathrm{O}_{3}-450{ }^{\circ} \mathrm{C}$ & 22,5 & 21,5 & 0,8 \\
$\mathrm{Al}_{2} \mathrm{O}_{3}-500^{\circ} \mathrm{C}$ & 26,2 & 24,2 & 2,0 \\
\hline $\mathrm{V} / \mathrm{Al}-450^{\circ} \mathrm{C}$ & 10,3 & 8,8 & 1,5 \\
$\mathrm{~V} / \mathrm{Al}-500{ }^{\circ} \mathrm{C}$ & 7,2 & 6,1 & 0,9 \\
\hline $\mathrm{VK} / \mathrm{Al}-450^{\circ} \mathrm{C}$ & 4,2 & 3,7 & 0,3 \\
$\mathrm{VK} / \mathrm{Al}-500^{\circ} \mathrm{C}$ & 4,5 & 4,1 & 0,4 \\
\hline
\end{tabular}

Os resultados da atividade catalítica, obtidos na reação de decomposição do isopropanol, na temperatura de $250{ }^{\circ} \mathrm{C}$ são mostrados na Tabela 2 . A impregnação do suporte com vanádio diminuiu a taxa de reação e a produção de propeno em ambas as temperaturas de calcinação. Em contrapartida, houve um aumento na produção de éter nos catalisadores calcinados a $450{ }^{\circ} \mathrm{C}$ e uma diminuição nos calcinados a $500{ }^{\circ} \mathrm{C}$. Já na co-impregnação com vanádio e potássio observou-se uma diminuição na taxa de reação e na produção de propeno, e a produção de éter foi praticamente desprezível. Este resultado se deve à acidez do suporte ter sido inibida pela presença do metal alcalino devido a um bloqueio sobre os sítios ácidos e, consequentemente, na distribuição da força ácida da alumina pela presença do mesmo.

\section{CONCLUSÕES}

As propriedades texturais do suporte $\mathrm{Al}_{2} \mathrm{O}_{3}$-X foram modificadas pela adição de vanádio e potássio, efeito esse atribuído ao bloqueio dos microporos por essas espécies. No DRX o precursor revelou um material composto por boehmita e baierita. Os catalisadores calcinados às temperaturas de 450 e $500{ }^{\circ} \mathrm{C}$ impregnados com vanádio e co-impregnados com vanádio e potássio apresentaram apenas as $\gamma-\mathrm{Al}_{2} \mathrm{O}_{3}$ e $\eta-\mathrm{Al}_{2} \mathrm{O}_{3}$. Nos resultados de RTP, os catalisadores apresentaram um único pico de redução do vanádio. A partir da reação de decomposição do isopropanol, conclui-se que os catalisadores apresentaram basicamente sítios ácidos, evidenciado pela formação de propeno e éter diisopropílico. Além disso, verificou-se que a adição de vanádio e potássio ao suporte promoveu uma diminuição da atividade catalítica.

\section{AGRADECIMENTOS}

Ao LCP-INPE de Cachoeira Paulista/SP e à FAPESP (2013/23400-4).

\section{REFERENCIAS}

CORTEZ, G.G.; FIERRO, J.L.G.; BAÑARES, M.A., Role of potassium on the structure and activity of alumina-supported vanadium oxide catalysts for propane oxidative dehydrogenation. Catal. Today, v. 78, p. 219-228, 2003.

ERMINI, V.; FINOCCHIO E.; SECHI S.; BUSCA, G.; ROSSINI S., Propane oxydehydrogenation over alumina-supported vanadia doped with manganese and potassium. Appl. Catal., v. 198, p. 67-79, 2000.

JUAN-JUAN, J.; ROMÁN-MARTÍNEZ, M. C.; ILLÁN-GÓMEZ, M. J., Effect of potassium content in the activity of $\mathrm{K}$-promoted $\mathrm{Ni} / \mathrm{Al}_{2} \mathrm{O}_{3}$ catalysts for the dry reforming of methane. Appl. Catal., v. 301, p. 9-15, 2006. 
KORANNE, M.M.; GOODWIN, J. G.; MARCELIN, G. Characterization of silica- and alumina-supported vanadia catalysts using temperature programmed reduction. $J$. Catal., v. 148, p. 369-377, 1994.

SCHOLZ, J.; WALTER, A.; RESSLER, T., Influence of MgO-modified SBA-15 on the structure and catalytic activity of supported vanadium oxide catalysts. J. Catal., v.309, p. 105-114, 2013.

TEIXEIRA, V. G.; COUTINHO, F. M. B.; GOMES, A. S. Principais métodos de caracterização da porosidade de resinas à base de divinilbenzeno. Quim. Nova, v. 24(6), p. 808-818, 2001. 